\begin{tabular}{|c|l|}
\hline Title & Construction of a Weyl Representation from a Weak Weyl Representation of the Canonical Commutation Relation \\
\hline Author(s) & A rai, A sao; Matsuzawa, Y asumichi \\
\hline Citation & $\begin{array}{l}\text { Letters in Mathematical Physics, 83(2), 201-211 } \\
\text { https:/doi.org/10.1007/311005-008-0220-4 }\end{array}$ \\
\hline Issue Date & 2008-02 \\
\hline Doc URL & http://hdl.handle.net/2115/32612 \\
\hline Rights & The original publication is available at www.springerlink.com \\
\hline Type & article (author version) \\
\hline File Information & ArMa.pdf \\
\hline
\end{tabular}

Instructions for use 


\title{
Construction of a Weyl Representation from a Weak Weyl Representation of the Canonical Commutation Relation
}

\author{
Asao Arai* $^{*}$ and Yasumichi Matsuzawa ${ }^{2}$ \\ Department of Mathematics, Hokkaido University \\ Sapporo 060-0810 \\ Japan \\ ${ }^{1}$ E-mail: arai@math.sci.hokudai.ac.jp \\ ${ }^{2}$ E-mail: s073035@math.sci.hokudai.ac.jp
}

\begin{abstract}
Weak Weyl representations of the canonical commutation relation (CCR) with one degree of freedom are considered in relation to the theory of time operator in quantum mechanics. It is proven that there exists a general structure through which a weak Weyl representation can be constructed from a given weak Weyl representation. As a corollary, it is shown that a Weyl representation of the CCR can be constructed from a weak Weyl representation which satisfies some additional property. Some examples are given.
\end{abstract}

Mathematics Subject Classification (2000). 81Q10, 47N50.

Keywords. canonical commutation relation, weak Weyl representation, Weyl representation, Hamiltonian, time operator, spectrum.

\section{Introduction}

Let $H$ be a self-adjoint operator on a complex Hilbert space $\mathcal{H}$ and $T$ be a symmetric operator on $\mathcal{H}$. We say that the pair $(T, H)$ obeys the weak Weyl relation if, for all $t \in \mathbb{R}$, $e^{-i t H} \mathcal{D}(T) \subset \mathcal{D}(T)(\mathcal{D}(T)$ denotes the domain of $T)$ and

$$
T e^{-i t H} \psi=e^{-i t H}(T+t) \psi, \quad \forall \psi \in \mathcal{D}(T) .
$$

From the representation theoretic point of view, the pair $(T, H)$ is called a weak Weyl representation of the canonical commutation relation (CCR) with one degree of freedom

${ }^{*}$ Corresponding author. The work is supported by the Grant-in-Aid No.17340032 for Scientific Research from Japan Society for the Promotion of Science (JSPS). 
[3]. This comes from the following facts: (i) every pair $(T, H)$ obeying the weak Weyl relation satisfies the CCR:

$$
[T, H] \psi=i \psi, \quad \forall \psi \in \mathcal{D}(T H) \cap \mathcal{D}(H T),
$$

i.e., $(T, H)$ is a representation of the $\mathrm{CCR}$ with one degree of freedom, but the converse is not true ; (ii) every weak Weyl representation $(T, H)$ with $T$ self-adjoint is a Weyl representation of the CCR, i.e., it satisfies the Weyl relation

$$
e^{i t T} e^{i s H}=e^{-i s t} e^{i s H} e^{i t T}, \quad s, t \in \mathbb{R}
$$

[6, Proposition 2.1-(iii)].

Detailed analyses of weak Weyl representations were first given by Schmüdgen $[9,10]$ (in the notation there, $T=P, H=-Q)$. Then Miyamoto [6] used the weak Weyl relation to develop a theory of time operator in quantum mechanics. In the context where $H$ is the Hamiltonian of a quantum system, the operator $T$ is called a time operator with respect to (w.r.t.) $H$ [6]. Motivated by this work, one of the present authors extended the framework of the theory of time operator to a more general one, introducing a notion of generalized time operators [3]. We use the term "time operator" in the general context too. Along this line of research, spectral analysis for time operators has been made in [4].

This paper is a continuation of the previous work [3, 4]. The main purpose of it is to point out that there exists a remarkable structure through which a Weyl representation can be constructed from a weak Weyl representation which satisfies some additional property. This structure, which may have physical significance too, makes it possible, for example, to identify the spectrum $\sigma(|H|)$ of $|H|$ (the absolute value of $H$ ) as $\sigma(|H|)=[0, \infty)$ under some condition.

The present paper is organized as follows. In Section 2, we state the fundamental result of the present paper as Theorem 2.4, which tells us that every weak Weyl representation of the CCR produces another weak Weyl representation of the CCR, and prove three corollaries of it, one of which (Corollary 2.6) is concerned with the construction of a Weyl representation of the CCR from a weak Weyl representation of the CCR as mentioned in the preceding paragraph. Section 3 is devoted to proof of Theorem 2.4. In the last section, we discuss some examples illustrating the abstract general results established in Section 2.

\section{Main Results}

Before stating the main results of this paper, we first recall some known facts.

The following fact is an interesting property to be kept in mind on weak Weyl representations:

Lemma 2.1 Let $(T, H)$ be a weak Weyl representation of the CCR on the Hilbert space $\mathcal{H}$. Suppose that $H$ is semi-bounded (i.e., bounded below or bounded above).

(i) ([3, Theorem 2.8]) Then $T$ is not self-adjoint. 
(ii) ([4, Theorem 2.1-(i)]) If $H$ is bounded below, then the spectrum $\sigma(T)$ of $T$ is either $\mathbb{C}$ (the set of complex numbers) or $\bar{\Pi}_{+}:=\{z \in \mathbb{C} \mid \Im z \geq 0\}$, the closed upper half-plane of $\mathbb{C}$.

(iii) ([4, Theorem 2.1-(ii)]) If $H$ is bounded above, then $\sigma(T)$ is either $\mathbb{C}$ or $\bar{\Pi}_{-}:=$ $\{z \in \mathbb{C} \mid \Im z \leq 0\}$, the closed lower half-plane of $\mathbb{C}$.

(iv) ([4, Theorem 2.1-(iii)]) If $H$ is bounded, then $\sigma(T)=\mathbb{C}$.

For a closable linear operator $C$ on a Hilbert space, we denote its closure by $\bar{C}$. Let $(T, H)$ be a weak Weyl representation of the CCR on the Hilbert space $\mathcal{H}$. Then it is easy to see that $(\bar{T}, H)$ also is a weak Weyl representation of the CCR. Hence, without loss of generality, one can assume that $T$ is closed. In what follows we take this assumption, unless otherwise stated.

Lemma 2.2 ([6, Proposition 2.1]) There exists a dense subspace $\mathcal{D} \subset \mathcal{H}$ such that $\mathcal{D} \subset$ $\mathcal{D}(T H) \cap \mathcal{D}(H T)$ and $H \mathcal{D} \subset \mathcal{D}$.

This proposition implies that $\mathcal{D}(T H) \cap \mathcal{D}(H T)$ is dense in $\mathcal{H}$. Hence one can consider the adjoint $(H T)^{*}\left(\operatorname{resp} .(T H)^{*}\right)$ of $H T(\operatorname{resp} . T H)$ with $(H T)^{*} \supset T H\left(\operatorname{resp} .(T H)^{*} \supset\right.$ $H T)$, which implies that $\mathcal{D}\left((H T)^{*}\right)$ (resp. $\left.\mathcal{D}\left((T H)^{*}\right)\right)$ is dense in $\mathcal{H}$. Hence $H T$ and $T H$ are closable and

$$
(T H)^{*} \supset \overline{H T}, \quad(H T)^{*} \supset \overline{T H} .
$$

Therefore the linear operator

$$
D:=\frac{1}{2}(T H+\overline{H T})
$$

on $\mathcal{H}$ is a symmetric operator.

The following result also is known:

Lemma 2.3 ([9, Corollary 2], [6, Theorem 4.4]) The self-adjoint operator $H$ is purely absolutely continuous.

This result implies, in particular, that the point spectrum $\sigma_{\mathrm{p}}(H)$ (the set of all the eigenvalues of $H$ ) is empty. In particular, 0 is not an eigenvalue of $H$. Hence, via the functional calculus, we can define a self-adjoint operator

$$
L:=\log |H| .
$$

The first of the main results of this paper is as follows:

Theorem 2.4 For all $t \in \mathbb{R}, e^{-i t L} \mathcal{D}(D)=\mathcal{D}(D)$ and

$$
D e^{-i t L}=e^{-i t L}(D+t) .
$$

This theorem tells us that the pair $(D, L)$ is a weak Weyl representation of the CCR. Therefore Theorem 2.4 reveals a general structure or mechanism through which every weak Weyl representation produces another weak Weyl representation.

Theorem 2.4 yields some corollaries. 
Corollary 2.5 Suppose that $|H| \geq c$ or $|H| \leq c$ for some constant $c>0$. Then $D$ is not essentially self-adjoint. Moreover the following (i)-(iii) hold:

(i) If $|H| \geq c$, then $\sigma(D)$ is either $\mathbb{C}$ or $\bar{\Pi}_{+}$.

(ii) If $|H| \leq c$, then $\sigma(D)$ is either $\mathbb{C}$ or $\bar{\Pi}_{-}$.

(iii) If $|H|$ is bounded with $0<c_{1} \leq|H| \leq c_{2}$ for some positive constants $c_{1}$ and $c_{2}$, then $\sigma(D)=\mathbb{C}$.

Proof. Under the assumption that $|H| \geq c>0$ (resp. $|H| \leq c)$, we have $L \geq \log c$ (resp. $L \leq \log c$ ). Hence $L$ is semi-bounded. Therefore, by Lemma 2.1-(i), $D$ is not essentially self-adjoint.

(i) If $|H| \geq c$, then $L$ is bounded below as shown above. Hence, by Lemma 2.1-(ii), $\sigma(D)$ is either $\mathbb{C}$ or $\bar{\Pi}_{+}$.

(ii) If $|H| \leq c$, then $L$ is bounded above as already seen. Hence, by Lemma 2.1-(ii), $\sigma(D)$ is either $\mathbb{C}$ or $\bar{\Pi}_{-}$. $\mathbb{C}$.

(iii) Under the present assumption, $L$ is bounded. Hence, by Lemma 2.1-(iii), $\sigma(D)=$

The next corollary is concerned with a construction of a Weyl representation of the CCR from a weak Weyl representation of the CCR:

Corollary 2.6 Suppose that D is essentially self-adjoint. Then

$$
e^{i s \bar{D}} e^{i t L}=e^{-i s t} e^{i t L} e^{i s \bar{D}}
$$

Namely $(\bar{D}, L)$ is a Weyl representation of the CCR.

Proof. By (2.4), we have $e^{i t L} \bar{D} e^{-i t L}=\bar{D}+t(\forall t \in \mathbb{R})$, which, together with the functional calculus for the self-adjoint operator $\bar{D}$, implies (2.5).

Remark 2.1 In view of Corollary 2.6, it is very important and interesting to find an additional condition for the weak Weyl representation $(T, H)$ of the CCR under which $D$ is essentially self-adjoint. But, in this paper, we do not discuss this problem.

Let $q$ and $p$ be the operators on the Hilbert space $L^{2}(\mathbb{R})$ defined by

$$
q:=M_{x}, \quad p:=-i D_{x}
$$

where $M_{x}$ is the multiplication operator by the variable $x \in \mathbb{R}$ and $D_{x}$ is the generalized differential operator in $x$. It is well known that $q$ and $p$ are self-adjoint and $(q, p)$ is a Weyl representation of the CCR. The representation $(q, p)$ of the CCR is called the Schrödinger representation of the CCR.

Corollary 2.7 Let $\mathcal{H}$ be separable. Suppose that D is essentially self-adjoint. Then there exist mutually orthogonal closed subspaces $\mathcal{H}_{n}$ of $\mathcal{H}$ such that the following (i)-(iii) hold:

(i) $\mathcal{H}=\oplus_{n=1}^{N} \mathcal{H}_{n}$ (N a positive integer or $\infty$ ), 
(ii) For each $n$ and all $t \in \mathbb{R}, e^{i t \bar{D}}: \mathcal{H}_{n} \rightarrow \mathcal{H}_{n}, e^{i t L}: \mathcal{H}_{n} \rightarrow \mathcal{H}_{n}$,

(iii) For each $n$, there exists a unitary operator $U_{n}: \mathcal{H}_{n} \rightarrow L^{2}(\mathbb{R})$ such that

$$
U_{n} \bar{D} U_{n}^{-1}=q, \quad U_{n} L U_{n}^{-1}=p .
$$

In particular

$$
\begin{aligned}
& \sigma(|H|)=[0, \infty), \\
& \sigma(\bar{D})=\mathbb{R}, \quad \sigma_{\mathrm{p}}(\bar{D})=\emptyset .
\end{aligned}
$$

Proof. By Corollary 2.6, $(\bar{D}, L)$ is a Weyl representation of the CCR. Since $\mathcal{H}$ is separable by the present assumption, the first half of Corollary 2.7 follows from the von Neumann uniqueness theorem ([7], [8, Theorem VIII.14]). Hence we have

$$
\begin{aligned}
& \sigma(L)=\sigma(p)=\mathbb{R}, \quad \sigma_{\mathrm{p}}(L)=\sigma_{\mathrm{p}}(p)=\emptyset, \\
& \sigma(\bar{D})=\sigma(q)=\mathbb{R}, \quad \sigma_{\mathrm{p}}(\bar{D})=\sigma_{\mathrm{p}}(q)=\emptyset .
\end{aligned}
$$

By the spectral mapping theorem, we have (2.7).

Remark 2.2 By Lemma 2.3, $\sigma_{\mathrm{p}}(|H|)=\emptyset$.

Remark 2.3 The Schrödinger representation $(q, p)$ is viewed as a special realization of $(T, H)$, for example, $(T, H)=(q, p)$ (or $(T, H)=(p,-q))$. In this case we have

$$
\begin{aligned}
& L=\log |p|, \\
& D=D_{\mathrm{S}}:=\frac{1}{2}(q p+\overline{p q}) .
\end{aligned}
$$

It is well known (e.g., [5, p.63]) that $\bar{D}_{\mathrm{S}}$ is the generator of the strongly continuous one-parameter unitary group $\{u(\theta)\}_{\theta \in \mathbb{R}}$ of dilation on $L^{2}(\mathbb{R})$ defined by

$$
(u(\theta) f)(x)=e^{\theta / 2} f\left(e^{\theta} x\right), \quad f \in L^{2}(\mathbb{R}), \text { a.e. } x \in \mathbb{R},
$$

i.e., $\bar{D}_{\mathrm{S}}$ is a self-adjoint operator such that

$$
u(\theta)=e^{i \theta \bar{D}_{\mathrm{S}}}, \quad \theta \in \mathbb{R} .
$$

For an open set $\Omega$ of $\mathbb{R}$, we denote by $C_{0}^{\infty}(\Omega)$ the set of infinitely differentiable functions on $\Omega$ with compact support in $\Omega$.

Since $C_{0}^{\infty}(\mathbb{R}) \subset \mathcal{D}\left(D_{\mathrm{S}}\right)$ and each $u(\theta)$ leaves $C_{0}^{\infty}(\mathbb{R})$ invariant, it follows from a general theorem (e.g., [8, Theorem VIII.10]) that $D_{\mathrm{S}}$ is essentially self-adjoint on $C_{0}^{\infty}(\mathbb{R})$. Thus the present example is a trivial illustration of Corollary 2.7. 


\section{Proof of Theorem 2.4}

We recall an important formula:

Lemma 3.1 ([9, Proposition 1], [3, Theorem 6.2-(i)]) Let $f$ be a continuously differentiable function on $\mathbb{R}$ such that $f$ and $f^{\prime}$ are bounded on $\mathbb{R}$. Then $f(H) \mathcal{D}(T) \subset \mathcal{D}(T)$ and

$$
T f(H) \psi-f(H) T \psi=i f^{\prime}(H) \psi, \quad \psi \in \mathcal{D}(T) .
$$

We now go into proof of Theorem 2.4. For $t \in \mathbb{R}$ and $\varepsilon>0$, we define the function $f_{\varepsilon}$ on $\mathbb{R}$ by

$$
f_{\varepsilon}(\lambda):=e^{-i t \log \sqrt{\lambda^{2}+\varepsilon^{2}}},
$$

It is easy to see that $f_{\varepsilon}$ is a continuously differentiable bounded function on $\mathbb{R}$ and $f_{\varepsilon}^{\prime}$ is bounded on $\mathbb{R}$ with

$$
\left|f_{\varepsilon}(\lambda)\right|=1, \quad f_{\varepsilon}^{\prime}(\lambda)=-\frac{i t \lambda}{\lambda^{2}+\varepsilon^{2}} f_{\varepsilon}(\lambda)
$$

Hence we can apply Lemma 3.1 to obtain that

$$
T f_{\varepsilon}(H) \psi-f_{\varepsilon}(H) T \psi=i f_{\varepsilon}^{\prime}(H) \psi, \quad \psi \in \mathcal{D}(T) .
$$

Let $\phi \in \mathcal{D}(T H)$. Then $H \phi \in \mathcal{D}(T)$. Hence

$$
T f_{\varepsilon}(H) H \phi-f_{\varepsilon}(H) T H \phi=i f_{\varepsilon}^{\prime}(H) H \phi .
$$

It follows from the functional calculus that

$$
\lim _{\varepsilon \rightarrow 0} i f_{\varepsilon}^{\prime}(H) H \phi=t e^{-i t L} \phi, \quad \lim _{\varepsilon \rightarrow 0} f_{\varepsilon}(H) T H \phi=e^{-i t L} T H \phi, \quad \lim _{\varepsilon \rightarrow 0} f_{\varepsilon}(H) H \phi=H e^{-i t L} \phi .
$$

By the closedness of $T$, we conclude that $H e^{-i t L} \phi \in \mathcal{D}(T)$ and

$$
T H e^{-i t L} \phi=e^{-i t L}(T H+t) \phi, \quad \phi \in \mathcal{D}(T H) .
$$

For all $\eta \in \mathcal{D}(H)$ and $\psi \in \mathcal{D}(H T)$, we have by (3.2)

$$
\left\langle H \eta, T f_{\varepsilon}(H) \psi\right\rangle=\left\langle\eta, f_{\varepsilon}(H) H T \psi\right\rangle+\left\langle\eta, i H f_{\varepsilon}^{\prime}(H) \psi\right\rangle .
$$

Hence $T f_{\varepsilon}(H) \psi \in \mathcal{D}(H)$ and

$$
H T f_{\varepsilon}(H) \psi=f_{\varepsilon}(H) H T \psi+i H f_{\varepsilon}^{\prime}(H) \psi .
$$

Hence

$$
\lim _{\varepsilon \rightarrow 0} \operatorname{HTf}_{\varepsilon}(H) \psi=e^{-i t L} H T \psi+t e^{-i t L} \psi .
$$

Therefore $e^{-i t L} \psi \in \mathcal{D}(\overline{H T})$ and

$$
\overline{H T} e^{-i t L} \psi=e^{-i t L}(H T+t) \psi, \quad \psi \in \mathcal{D}(H T) .
$$


By a simple limiting argument, one can extend this result to all $\psi \in \mathcal{D}(\overline{H T})$ with $e^{-i t L} \mathcal{D}(\overline{H T})=\mathcal{D}(\overline{H T})$ and

$$
\overline{H T} e^{-i t L} \psi=e^{-i t L}(\overline{H T}+t) \psi, \quad \psi \in \mathcal{D}(\overline{H T}) .
$$

It follows from (3.3) and (3.4) that

$$
e^{-i t L} \mathcal{D}(T H) \cap \mathcal{D}(\overline{H T})=\mathcal{D}(T H) \cap \mathcal{D}(\overline{H T})
$$

and, for all $\psi \in \mathcal{D}(\overline{H T}) \cap \mathcal{D}(T H)$,

$$
D e^{-i t L} \psi=e^{-i t L}(D+t) \psi .
$$

Thus the desired results follow.

\section{Examples}

\subsection{Quantum mechanics on the half-line $(0, \infty)$}

Let $\mathbb{R}_{+}:=\{\lambda \mid \lambda>0\}=(0, \infty)$ and define the operators $H$ and $T$ on $L^{2}\left(\mathbb{R}_{+}\right)$as follows:

$$
H:=\hat{\lambda}_{+}:=M_{\lambda}, \quad T:=-\hat{p}_{+}:=i \frac{d}{d \lambda}, \mathcal{D}\left(\hat{p}_{+}\right):=C_{0}^{\infty}\left(\mathbb{R}_{+}\right)
$$

Then it is easy to see that $\hat{\lambda}_{+}$is self-adjoint, $-\hat{p}_{+}$is symmetric and $\left(-\hat{p}_{+}, \hat{\lambda}_{+}\right)$is a weak Weyl representation of the CCR. As already pointed out in [4, Example 2.1], one has

$$
\sigma\left(\hat{\lambda}_{+}\right)=[0, \infty), \quad \sigma\left(-\hat{p}_{+}\right)=\bar{\Pi}_{+} .
$$

In the present case we have

$$
\begin{aligned}
& L=\log |H|=B:=\log \hat{\lambda}_{+}, \\
& D=A:=-\frac{1}{2}\left(\hat{p}_{+} \hat{\lambda}_{+}+\overline{\hat{\lambda}_{+} \hat{p}_{+}}\right) .
\end{aligned}
$$

Lemma 4.1 The operator $A$ is essentially self-adjoint on $C_{0}^{\infty}\left(\mathbb{R}_{+}\right)$. Moreover

$$
\left(e^{-i \theta \bar{A}} f\right)(\lambda)=e^{\theta / 2} f\left(e^{\theta} \lambda\right), \quad f \in L^{2}\left(\mathbb{R}_{+}\right) .
$$

Proof. Similar to the proof of the fact stated in Remark 2.3.

Remark 4.1 Every unitary operator $u(\theta)$ mentioned in Remark 2.3 is reduced by $L^{2}\left(\mathbb{R}_{+}\right)$. We denote its reduced part by $u_{+}(\theta)$. Then $\left\{u_{+}(\theta)\right\}_{\theta \in \mathbb{R}}$ gives a unitary representation on $L^{2}\left(\mathbb{R}_{+}\right)$of the dilation group $\left\{e^{\theta}\right\}_{\theta \in \mathbb{R}}$ acting on $\mathbb{R}_{+}$. The lemma shows that $-\bar{A}$ is the generator of this unitary group. 
By Lemma 4.1 and Corollary 2.7, $(A, B)$ is unitarily equivalent to a direct sum of the Schrödinger representation $(q, p)$.

It may be instructive to find explicitly the unitary operator implementing the equivalence. Indeed this is possible as done below (cf. [2, Appendix B]).

We denote by $\mathcal{F}$ the Fourier transform on $L^{2}(\mathbb{R})$ :

$$
(\mathcal{F} u)(k):=\frac{1}{\sqrt{2 \pi}} \int_{\mathbb{R}} e^{-i k x} u(x) d x, \quad u \in L^{2}(\mathbb{R}), \text { a.e. } k \in \mathbb{R},
$$

in the $L^{2}$-sense. For each $f \in L^{2}\left(\mathbb{R}_{+}\right)$, we define $f_{e} \in L^{2}(\mathbb{R})$ by

$$
f_{e}(k):=f\left(e^{k}\right) e^{k / 2}, \quad \text { a.e. } k \in \mathbb{R}
$$

and introduce $E: L^{2}\left(\mathbb{R}_{+}\right) \rightarrow L^{2}(\mathbb{R})$ by

$$
E f:=f_{e}, \quad f \in L^{2}\left(\mathbb{R}_{+}\right) .
$$

Then $E$ is unitary. Using $E$ and $\mathcal{F}$, we define an operator $M: L^{2}\left(\mathbb{R}_{+}\right) \rightarrow L^{2}(\mathbb{R})$ by

$$
M:=\mathcal{F}^{-1} E .
$$

Obviously $M$ is unitary. Explicitly we have

$$
(M f)(x)=\frac{1}{\sqrt{2 \pi}} \int_{\mathbb{R}} f(\lambda) \lambda^{\left(\frac{1}{2}+i x\right)-1} d \lambda, \quad x \in \mathbb{R},
$$

for all $f \in L^{2}\left(\mathbb{R}_{+}\right)$such that

$$
\int_{\mathbb{R}_{+}} \frac{|f(\lambda)|}{\sqrt{\lambda}} d \lambda<\infty
$$

Hence $M$ is a Mellin transform. It is not so difficult to show that

$$
M A M^{-1}=q, \quad M B M^{-1}=p .
$$

For every constant $m>0$, the pair $\left(-\hat{p}_{+}, \hat{\lambda}_{+}+m\right)$ is obviously a weak Weyl representation. In this case we have $\hat{\lambda}_{+}+m \geq m>0$. Hence, by Corollary 2.5, the operator

$$
A_{m}:=-\frac{1}{2}\left(\hat{p}_{+}\left(\hat{\lambda}_{+}+m\right)+\overline{(\hat{\lambda}+m) \hat{p}_{+}}\right)
$$

is not essentially self-adjoint. This is an interesting phenomenon too (one can prove this fact directly by computing the deficiency indices of $A_{m}$ ). Moreover we can show that $\sigma\left(A_{m}\right)=\bar{\Pi}_{+}$(the proof is similar to that of [4, Theorem 4.2], but, in the present case, it is very easy). 


\subsection{The free Hamiltonian of a non-relativistic quantum particle in $\mathbb{R}$ and the Aharonov-Bohm time operator}

Let $(q, p)$ be the Schrödinger representation of the CCR with one degree of freedom as in (2.6). For a constant $m>0$, we define

$$
H_{0}:=\frac{1}{2 m} p^{2}=-\frac{1}{2 m} \Delta_{x}
$$

acting in $L^{2}(\mathbb{R})$, where $\Delta_{x}:=D_{x}^{2}$. The operator $H_{0}$ is called the one-dimensional free Hamiltonian of a non-relativistic quantum particle with mass $m$. It is well known that $H_{0}$ is a non-negative self-adjoint operator on $L^{2}(\mathbb{R})$ and its spectrum is purely absolutely continuous with

$$
\sigma\left(H_{0}\right)=[0, \infty)
$$

Let

$$
T_{\mathrm{AB}}:=\frac{m}{2}\left(q p^{-1}+p^{-1} q\right)
$$

with domain

$$
\mathcal{D}\left(T_{\mathrm{AB}}\right):=\mathcal{F}^{-1} C_{0}^{\infty}(\mathbb{R} \backslash\{0\}) .
$$

It is easy to see that $T_{\mathrm{AB}}$ is a symmetric operator on $L^{2}(\mathbb{R})$. Moreover, $\left(T_{\mathrm{AB}}, H_{0}\right)$ is a weak Weyl representation of the CCR [6]. The operator $T_{\mathrm{AB}}$ is called the Aharonov-Bohm time operator [1]. By Lemma 2.1, $T_{\mathrm{AB}}$ is not essentially self-adjoint. It is proven in [4] that

$$
\sigma\left(T_{\mathrm{AB}}\right)=\bar{\Pi}_{+} .
$$

In the present example, we have

$$
\begin{aligned}
& L=P_{0}:=\log H_{0}, \\
& D=Q_{0}:=\frac{1}{2}\left(T_{\mathrm{AB}} H_{0}+\overline{H_{0} T_{\mathrm{AB}}}\right) \supset q_{0},
\end{aligned}
$$

with

$$
q_{0}:=\frac{1}{2} D_{\mathrm{S}} \mid \mathcal{D}\left(T_{\mathrm{AB}}\right)
$$

where $D_{\mathrm{S}}$ is given by (2.10). Therefore we can apply Corollary 2.7 to conclude that $\left(\bar{Q}_{0}, P_{0}\right)$ is unitarily equivalent to a direct sum of the Schrödinger representation $(q, p)$ of the CCR.

In fact one can prove explicitly that $\left(\bar{Q}_{0}, P_{0}\right)$ is unitarily equivalent to a two direct sum of the Schrödinger representation $(q, p)$ of the CCR. This is essentially done in $[2$, Appendix B]. Hence we omit the details.

\subsection{A relativistic free Hamiltonian and a time operator of it}

A one-dimensional relativistic free Hamiltonian of a quantum particle with mass $m \geq 0$ and without spin is given $\mathrm{y}$

$$
H(m):=\sqrt{p^{2}+m^{2}}
$$


acting in $L^{2}(\mathbb{R})$. A time operator w.r.t. $H(m)$ is defined by

$$
T(m):=\frac{1}{2}\left\{H(m) p^{-1} q+q p^{-1} H(m)\right\}
$$

with domain $\mathcal{D}(T(m)):=\mathcal{F}^{-1} C_{0}^{\infty}(\mathbb{R} \backslash\{0\})([3$, Example 11.4]). In this case we have

$$
\begin{aligned}
& L=P(m):=\log H(m), \\
& D=Q(m):=\frac{1}{2}(T(m) H(m)+\overline{H(m) T(m)}) \supset 2\left(D_{\mathrm{S}}+m T_{\mathrm{AB}}\right) .
\end{aligned}
$$

Hence the following results hold:

(i) Consider the case $m=0$. Then $Q(0)$ is essentially self-adjoint. Hence $(\overline{Q(0)}, P(0))$ is unitarily equivalent to a direct sum of the Schrödinger representation $(q, p)$.

(ii) If $m>0$, then $Q(m)$ is not essentially self-adjoint.

In case (i), we can show, as in the case of the preceding example, that $(\overline{Q(0)}, P(0))$ is unitarily equivalent to a two direct sum of the Schrödinger representation $(q, p)$.

In case (ii), we can prove that $\sigma(Q(m))=\bar{\Pi}_{+}$(the proof is similar to that of $[4$, Theorem 4.3]).

Remark 4.2 Results obtained in this section can be extended easily to higher dimensional versions of the examples.

\section{References}

[1] Y. Aharonov and D. Bohm, Time in the quantum theory and the uncertainty relation for time and energy, Phys. Rev. 122, 1649-1658(1961).

[2] A. Arai, Rigorous theory of spectra and radiation for a model in quantum electrodynamics, J. Math. Phys. 24, 1896-1910 (1983).

[3] A. Arai, Generalized weak Weyl relation and decay of quantum dynamics, Rev. Math. Phys. 17 , 1071-1109 (2005).

[4] A. Arai, Spectrum of time operators, Lett. Math. Phys. 80, 211-221 (2007).

[5] H. L. Cycon, R. G. Froese, W. Kirsch and B. Simon, Schrödinger Operators, Springer, Berlin Heidelberg, 1987.

[6] M. Miyamoto, A generalized Weyl relation approach to the time operator and its connection to the survival probability, J. Math. Phys. 42, 1038-1052 (2001).

[7] J. von Neumann, Die Eindeutigkeit der Schrödingerschen Operatoren, Math. Ann. 104, 570-578 (1931).

[8] M. Reed and B. Simon, Methods of Modern Mathematical Physics I: Functional Analysis, Academic Press, New York, 1972. 
[9] K. Schmüdgen, On the Heisenberg commutation relation. I, J. Funct. Anal. 50, 8-49 (1983).

[10] K. Schmüdgen, On the Heisenberg commutation relation. II, Publ. RIMS, Kyoto Univ. 19, 601-671 (1983). 